\title{
A method of drusen measurement based on reconstruction of fundus background reflectance
}

\author{
R T Smith, J K Chan, T Nagasaki, J R Sparrow, I Barbazetto
}

Br J Ophthalmol 2005;89:87-91. doi: 10.1136/bjo.2004.042937

See end of article for authors' affiliations

.....................

Correspondence to:

Dr R T Smith, Harkness Eye Institute Columbia University College of Pysicians and Surgeons, New York, NY10032, USA; rts1@columbia.edu

Accepted for publication 7 April 2004
Background: The hallmarks of age related macular degeneration (AMD) are the subretinal deposits known as drusen. Current manual methods of drusen segmentation and quantification are laborious and subjective. The authors introduced a digital method and tested it for accuracy and reliability.

Methods: Fourteen eyes with drusen were selected. The authors digitally reconstructed the macular background using normal background areas ("dots") fitted to quadratic polynomials in two zones. The model was used to level the reflectance for the purpose of segmenting drusen by a global threshold. Measurements of drusen areas were compared with those of a semi-automated background levelling technique and manual drawings from stereo pairs.

Results: Intraobserver reproducibility had standard deviations from $0.1 \%$ to $4.1 \%$. Interobserver reproducibility yielded $95 \%$ limits of agreement of $-2.7 \%$ to $6.3 \%$. The dots method compared with manual drawings and with the semi-automated method had $95 \%$ limits of agreement of $-8.3 \%$ to $2.8 \%$ and $-7.1 \%$ to $4.8 \%$, respectively.

Conclusions: The dots method was reproducible and accurate with respect to validated methods. It provided less total operating time and greater precision than that of standard fundus photo grading. With implementation of commercial software, this technique for macular image analysis has potential for use in clinical research.
C olour fundus photographs are routinely employed for diagnostic purposes and are central to clinical studies of macular disease. ${ }^{1-13}$ Multiple publications have shown that extensive drusen are strongly correlated with the progression of age related macular degeneration (AMD). ${ }^{2-10}$ The current standard for fundus photography grading in AMD is manual grading of stereo pairs at the light box. ${ }^{11}{ }^{12}$ It has served well in multicenter trials, ${ }^{13}$ but measurements are subjective, costly, and time consuming.

There has been continued interest in the use of digital techniques for quantification of drusen over the last two decades. ${ }^{14-19}$ Developing techniques that measure drusen area more accurately would improve the data being gathered in clinical trials-for example, the study of drusen resorption after laser photocoagulation. ${ }^{6-8}{ }^{20-23}$ However, digital methods have yet to gain wide acceptance and usually require some human supervision. ${ }^{1424}$

A major difficulty is that the normal background, on which the pathology is superimposed, has an inherently nonuniform reflectance. The normal background is less reflectant centrally, with radially increasing reflectance towards the arcades. This geometry, due in part to luteal pigment in the central macula (fovea and parafovea ${ }^{25} 26$ and analysed in detail in Smith et $a^{2^{27}}$ ) is superimposed on the underlying or "true" drusen reflectance. To correct this variable reflectance, we have previously developed a semi-automated digital method to level the macular background..$^{28}$ The background levelling process gradually raises the intensities of the green channel image in user defined oval regions. The background becomes uniform while drusen are simultaneously brightened for segmentation. This method was reproducible and correlated well with standard fundus grading. ${ }^{28}$

Our new method, which we call the "dots" method, replaces the interactive routine with a mathematical model ${ }^{27}$ that can automatically reconstruct and level the macular background. We had previously verified that the mathematical model using quadratic polynomials can fit normal fundus images accurately ${ }^{27}$ (mean absolute error $7.2 \%$ (SD $5.5 \%$ ) of net grey scale range for images modelled by dots). The quadratics were chosen because their level sets (defined by $\mathrm{Q}(\mathrm{x}, \mathrm{y})=$ constant $)$ are conic sections, of which ellipses are one, and our observation was that the foveal isobars defined by constant grey level most closely resembled ellipses. ${ }^{27}$ In this paper, we applied the reconstruction technique to maculas with drusen, and extended the model from the fovea to the larger $3000 \mu \mathrm{m}$ diameter subfield. The dots method was compared for accuracy and reproducibility to our semi-automated method and a gold standard technique of manual tracing with stereo slide viewing.

\section{METHODS \\ Subjects}

Fourteen cases with stage 2 or 3 age related maculopathy (as defined by the International Grading System $)^{11}$ were selected at random from the Columbia Macular Genetics Study (CMGS). The CMGS is an IRB approved study of the genetic variation in AMD at New York Presbyterian Hospital. Thus, all cases contained soft distinct and/or indistinct drusen with or without pigmentary abnormalities. All cases were more advanced than AREDS stage $2 .{ }^{13}$ All patients were white and over 60 years of age.

\section{Image acquisition and preparation}

Good quality stereo pairs of standard $35^{\circ}$ film based colour fundus photographs were scanned (CoolScan LS-2000, Nikon Corporation, Tokyo, Japan) at resolutions of 2700 pixels/inch. The images were saved as 24 bit RGB TIFF files.

All images were resized using Adobe Photoshop 5.5 (Adobe Systems Inc, San Jose, CA, USA) so that the distance from the centre of the macula to the temporal disc edge was approximately 500 pixels. This corresponded to an anatomical

Abbreviations: AMD, age related macular degeneration; CMGS, Columbia Macular Genetics Study 
distance of $3000 \mu \mathrm{m}$. A three channel correction for the large scale variation in brightness was used to standardise the image. ${ }^{28}$ The green channel, which contains the most detail and greatest contrast for drusen, was used for further processing. ${ }^{1428}$ A contrast enhanced, standardised image (Photoshop/Autolevels) was used for visual references.

A digital template defined by the Wisconsin grading system $^{12}$ was centred on the fovea. The regions studied were the central subfield of $1000 \mu \mathrm{m}$ diameter and the middle subfield between $1000 \mu \mathrm{m}$ and $3000 \mu \mathrm{m}$ diameter.

\section{Macular background reconstruction and drusen measurement}

We first applied a partial luteal correction to the green channel. ${ }^{28}$ The user then manually selected dots, $25 \mu \mathrm{m}$ squares of the remaining normal macular background, from the luteal corrected image. Figure IA shows an original fundus image with drusen and figure $1 \mathrm{~B}$ is a typical dots selection. The selected pixels (fig $1 \mathrm{C}$ ) plus the luteal corrected fundus image were then imported to Matlab 6.5 (Mathworks Inc, Natick, MA, USA), which computed a geometric model of the macular background. Briefly, we considered the pixel intensity values to be functions of their pixel coordinates $(x, y)$ in the $x-y$ plane. The general quadratic polynomial $\mathrm{q}(\mathrm{x}, \mathrm{y})=\mathrm{ax}^{2}+\mathrm{bxy}+\mathrm{cy^{2 }}+\mathrm{dx}+\mathrm{ey}+\mathrm{constant}$ in two variables was fit by a custom algorithm employing least squares methods to the given data set of pixel values. We optimised the six coefficients (a, b, c, d, e, constant) in each of the two zones, hence generating two quadratics q1 and q2. We smoothed boundary discontinuities in an annular transition zone (the area between $750 \mu \mathrm{m}$ diameter and $1000 \mu \mathrm{m}$ diameter) by an appropriate sigmoidal radial cubic spline.

The model is displayed as an elliptical contour map of grey scale intensity levels (fig lD). The model was subtracted from the luteal corrected fundus image to level the background, and brighten drusen deposits in areas where the background was darker (fig $1 \mathrm{E}$ and F). A constant offset ( 125 grey scale) was also added to make the resulting image visible. The precise choice of offset had no effect on final results. The drusen were segmented by a global threshold and superimposed onto the contrast enhanced image. The threshold was adjusted so that the boundaries of the segmented drusen objects and those of the contrast enhanced objects (fig lG and $\mathrm{H}$ ) achieved optimum subjective correspondence. For better results, we allowed different thresholds in the central and middle subfields. Drusen area as a percentage of the $3000 \mu \mathrm{m}$ diameter region was then quantified (Photoshop/ Histogram)

\section{Method reproducibility and validation}

Two independent graders used the dots method to measure drusen. An experienced retinal specialist graded the corresponding stereo slide pairs by a gold standard of digital manual tracing with stereo slide viewing. This is a more precise and digital analog of clinical stereo slide grading. The semi-automated method, previously validated, ${ }^{28}$ was also used for comparison.

To assess the robustness of the model to minimal dots selection, we limited the annular region to a well spaced selection of at most 10 dots per quadrant. In the central region, at least six dots were always used. This test was performed randomly on seven of the 14 images studied here. Drusen measurements were performed as before.

\section{Accuracy of model fit}

Another subset of seven images was chosen to assess the accuracy of the model fit to the residual luteal corrected background data after segmentation of drusen. The error data were obtained by subtracting the model fit from the luteal corrected image, excluding the segmented drusen and large retinal vessels. We then found the mean and standard deviation of the set of absolute values of the errors,
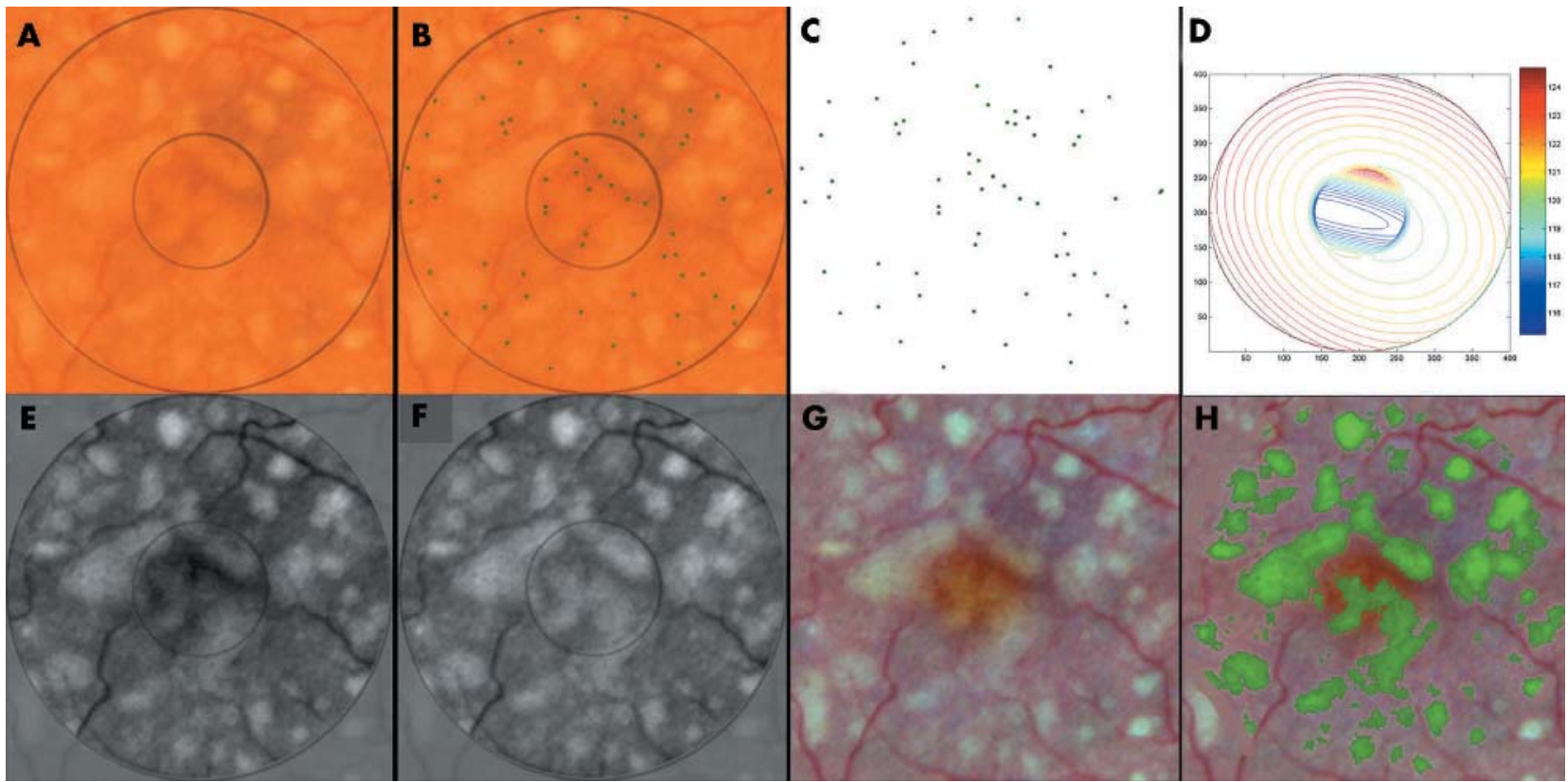

Figure 1 The dots method. (A) Original image. (B) Background dots selected from original. (C) The background dots (isolated) to which the quadratic polynomials are fit. (D) Contour graph of the two zone quadratic fit to the background points. Note that the fit varies more rapidly in the centre zone. The different colours correspond to the different levels of background intensity at each contour. (E) Green channel of the original image (grey scale, contrast enhanced in field of interest to visualise drusen). Note the darker background centrally. (F) Green channel (grey scale) of original image after subtracting the background quadratic fit functions, then contrast enhanced to show effect of background levelling and brightening of drusen. The background in this image is now uniform. (G) Full colour contrast enhanced version of the original image for ease of drusen visualisation. (H) Segmentation of the image in (F) by a single uniform threshold, then overlaid on (G) to show accuracy of segmentation. 
normalised as a percentage of the net grey scale range of the luteal corrected data (excluding drusen and retinal vessels). This was analogous to our previous measurements of the errors of the model fit to normal foveal data. ${ }^{27}$

\section{RESULTS}

Figure 2 shows drusen area measurements as a percentage of the $3000 \mu \mathrm{m}$ diameter zone obtained using the three methods. One grader performed measurements for all images twice using the dots model (fig 2A). The standard deviations ranged from $0.1 \%$ to $4.1 \%$. To show interobserver reproducibility, measurements were compared for two graders (fig 2B). The corresponding 95\% limits of agreement ${ }^{29}$ between measurements by the two graders ranged from $-2.7 \%$ to $6.3 \%$.

The mean absolute difference between the measurements by the dots method and the gold standard was 3.45\% (SD $1.88 \%$ ). The dots method was also compared with the semiautomated method with a mean absolute difference of $2.4 \%$ (SD 2.1\%). Measurements obtained by the dots method were compared with the gold standard and with our semiautomated method to yield 95\% limits of agreement of $-8.3 \%$ to $2.8 \%$ and $-7.1 \%$ to $4.8 \%$, respectively. Drusen measurements by the dots method were slightly lower in each case.

Figure 3A and D show an image analysed using a regular selection of dots and a reduced set of dots, respectively. The parameters that the algorithm calculated for the quadratic polynomials in both cases are shown in table 1. The contour maps created (fig 3B and E) are very similar, resulting in approximately the same levelling. Using the same threshold yielded drusen measurements of $32 \%$ area in both cases (fig 3C and F). In the seven images studied by the full set and by the reduced set, the mean absolute difference between measurements was $2.1 \%$ (SD $1.4 \%$ ).

The mean errors of the model fit to the residual background data ranged from $6.1 \%$ (SD $5.9 \%$ ) to $9.8 \%$ (SD 10.1\%) (median $7.2 \%($ SD $7.5 \%)$ ) as a percentage of the net image grey scale range (excluding drusen and large retinal vessels). Compared with our fits of normal images, ${ }^{27}$ the mean errors were quite comparable (median $7.2 \%$ for drusen images and $7.1 \%$ for normal images), but the standard deviations for the drusen images tended to be higher (median $7.5 \%$ for drusen images compared to $5.5 \%$ for normal images). The worst drusen background fit (mean error 9.8\%) was also worse than the worst normal image fit (mean error 8.7\%).

\section{DISCUSSION}

We modelled the geometry of the macular background (3000 $\mu \mathrm{m}$ diameter) in two zones. The method could have been extended to $6000 \mu \mathrm{m}$ diameter by adding additional zones. More dots would need to be selected but the same techniques would apply. A model with a single zone would perhaps have been simpler and eliminated the need for smoothing at boundary discontinuities. However, using separate zones had the advantage of separate fits for regions that had distinct luteal pigment densities. We also found that the pattern and orientation of the model varied with each image because the model was derived from the unique reflectance of the macula.

A key step in reconstruction of drusen laden maculas was that a small subset of dots could accurately model the full macular background. After drusen segmentation, the mean errors of the model fit to residual background found here were comparable to fits to normal data, ${ }^{27}$ but the standard deviations were higher. This suggests that even the residual

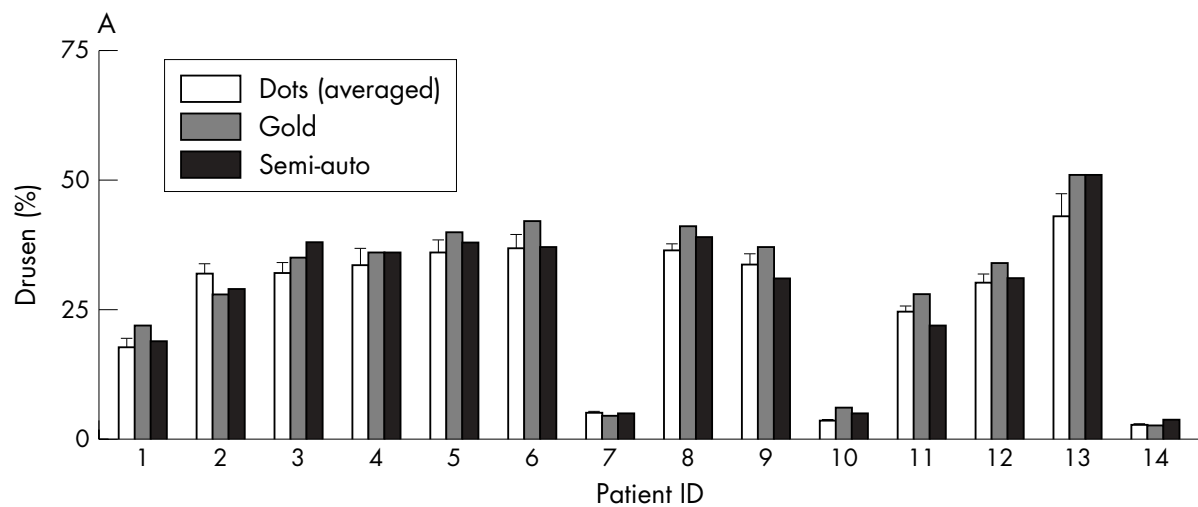

Patient ID
Figure 2 Comparison of dots, semiautomated, and gold standard methods. (A) Drusen area measurements as a percentage of the $3000 \mu \mathrm{m}$ diameter circle by the dots method compared with the semiautomated method and gold standard. Three independent trials of the dots method were averaged together, and the standard deviation is displayed to show reproducibility. (B) Comparison of the dots method with the semiautomated method and gold standard. The measurements with the dots method are displayed for two graders to compare interobserver reproducibility. Two independent trials by one grader were averaged to show intraobserver reproducibility.

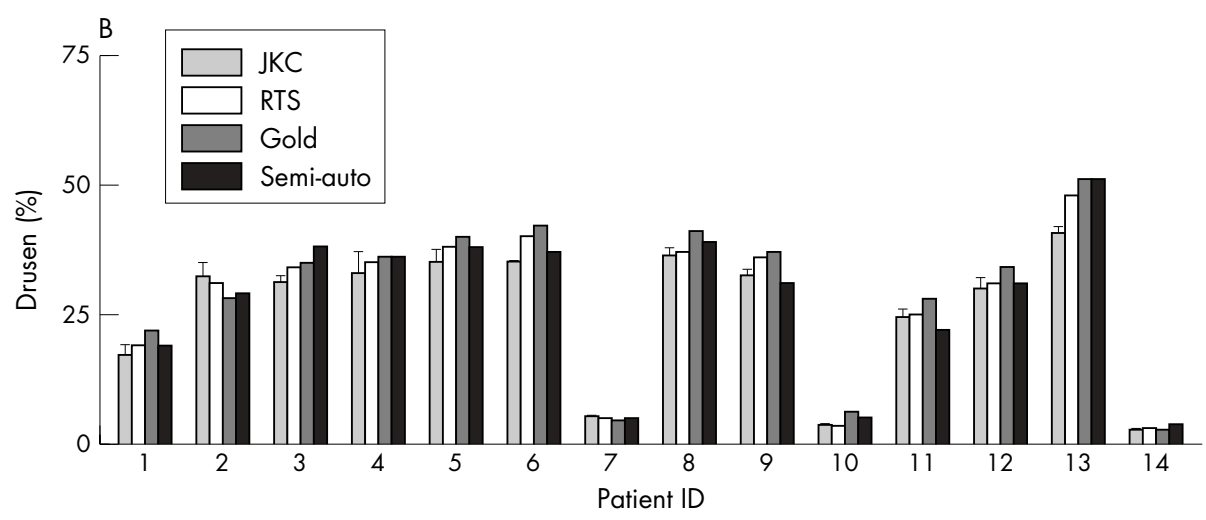




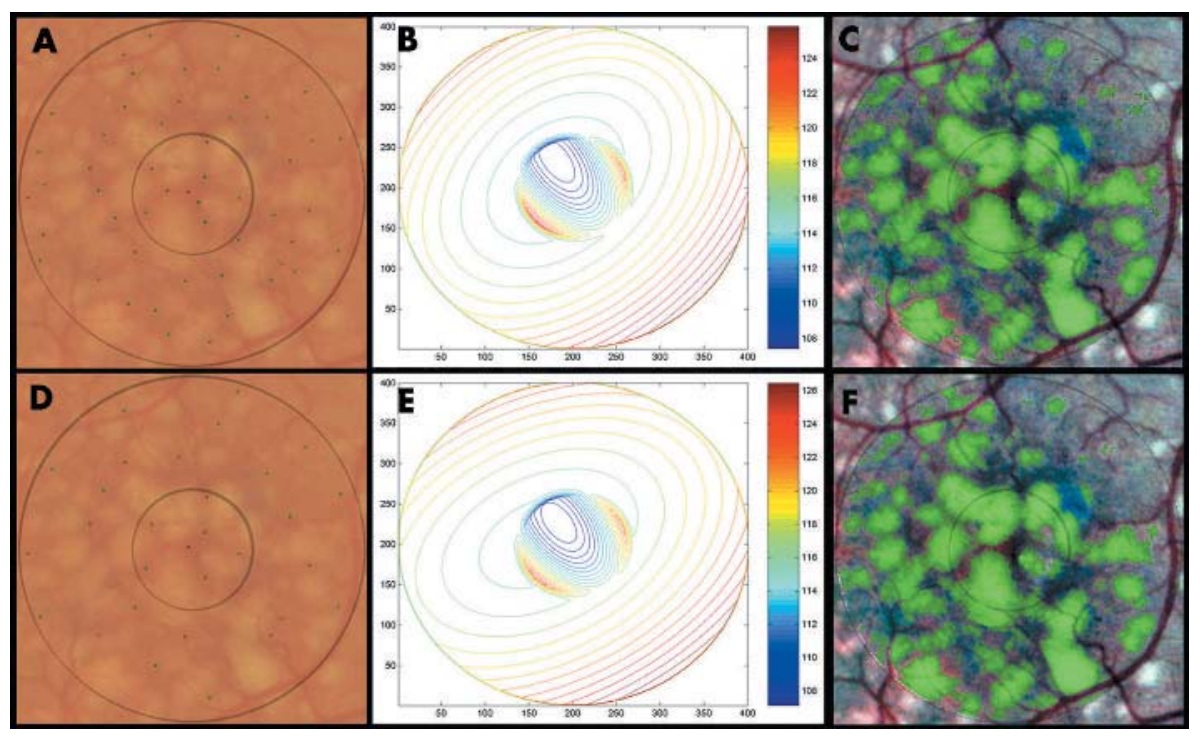

Figure 3 Results of minimum versus full dots selection. (A) Full dots selection (51 dots). (B) Contour graph of the two zone quadratic fit to these background dots. (C) Segmentation of the drusen, superimposed over the contrast enhanced fundus image. (D) A minimal dots selection (29 dots). (E) Contour graph based on these background dots. (F) Segmentation of the drusen, superimposed over the contrast enhanced fundus image.

"normal" background in a drusen image is more irregular than the background from a clinically normal eye.

We found that a selection of 6-8 dots per quadrant in the annular region yielded a model virtually identical to that obtained with 15 or more dots per quadrant. In the central region, with smaller area but greater intensity gradient, at least $6-8$ dots appeared to be a minimum. Hence, about 30-40 dots were sufficient. More importantly, the selection needed to be well distributed radially for proper background representation. However, drusen and vasculature precluded the use of any preset selection of dots. Manual identification of normal background is comparable to a trained grader looking for drusen. From a practical standpoint, a user trained to identify drusen can choose dots of background with efficiency and high accuracy. A major advantage of this method is that through the model a simple human intervention (dot selection) is leveraged into a precise segmentation that would be quite tedious to achieve by drawing drusen borders manually.

The semi-automated method, ${ }^{28}$ compared to the dots method, is potentially more accurate as its model is based on a series of ellipses that is more complex than a quadratic polynomial. However, the results in figure 2 showed that the semi-automated method did not differ significantly from the dots method in segmentation.

The two supervision steps-dots selection and threshold selection-each required about two minutes of user time. This compared with 10 minutes of operator time to draw a series of ellipses in the semi-automated method or about 20 minutes to make a gold standard drawing. Our method is

Table 1 Comparison of coefficients of the quadratic polynomial models in the full dots set and the reduced dots set

\begin{tabular}{|c|c|c|c|c|}
\hline \multirow[b]{2}{*}{ Coefficients } & \multicolumn{2}{|c|}{ Full dots selection } & \multicolumn{2}{|c|}{ Reduced dots selection } \\
\hline & $\begin{array}{l}\text { Central } \\
\text { zone }\end{array}$ & $\begin{array}{l}\text { Annular } \\
\text { zone }\end{array}$ & $\begin{array}{l}\text { Central } \\
\text { zone }\end{array}$ & $\begin{array}{l}\text { Annular } \\
\text { zone }\end{array}$ \\
\hline$A$ & 0.0020 & 0.0002 & 0.0023 & 0.0002 \\
\hline B & 0.0030 & -0.0001 & 0.0031 & -0.0001 \\
\hline C & 0.0033 & 0.0002 & 0.0035 & 0.0001 \\
\hline$D$ & -0.0740 & -0.0061 & -0.0682 & -0.0055 \\
\hline$E$ & 0.0296 & 0.0091 & 0.0365 & 0.0117 \\
\hline Constant & 107.96 & 114.22 & 107.33 & 114.88 \\
\hline
\end{tabular}

thus comparable to the time required for graders in the CMGS to do a semi-quantitative standard drusen grading.

The main source of variability_-selecting a threshold-was not significant based on our reproducibility statistic. The user determined visually the threshold that gave the best overall correspondence between the segmented image and the contrast enhanced image. Since the levelling brightens central drusen (see fig 1), the optimum threshold was easy to select. The subjectivity was comparable to deciding the boundaries in a gold standard drawing. In the future, we plan to automate the global threshold. One such technique is the Otsu method, ${ }^{30}$ which selects the optimal threshold based on a discriminant criterion that maximises the separability of two classes in grey levels. Adaptive histogram techniques have previously not been shown to perform well on drusen images, largely because of the variable macular background $^{1424}$ but they should perform well after the background has been levelled.

In summary, the dots method provided an image analysis tool that was reproducible, cost effective, and performed accurately with respect to both our semi-automated and gold standard methods. Implemented with commercially available software, the dots method is potentially useful for larger scale research on drusen in AMD.

\section{Authors' affiliations}

R T Smith, J K Chan, T Nagasaki, J R Sparrow, I Barbazetto, Department of Ophthalmology, Columbia University, New York, NY, USA

Funds for Columbia University Department of Ophthalmology from Research to Prevent Blindness (Drs Nagasaki and Sparrow). R T Smith, NY Community Trust. Takayuki Nagasaki, NIH EY00431. Janet R Sparrow, NIH EY12951.

\section{REFERENCES}

1 Klaver CC, Wolfs RC, Vingerling JR, et al. Age-specific prevalence and causes of blindness and visual impairment in an older population: the Rotterdam Study. Arch Ophthalmol 1998;116:653-8.

2 Smiddy WE, Fine SL. Prognosis of patients with bilateral macular drusen. Ophthalmology 1984:91:271-7.

3 Bressler SB, Maguire MG, Bressler NM, et al. Relationship of drusen and abnormalities of the retinal pigment epithelium to the prognosis of neovascular macular degeneration. The Macular Photocoagulation Study Group. Arch Ophthalmol 1990;108:1442-7.

4 Bressler NM, Bressler SB, Seddon JM, et al. Drusen characteristics in patients with exudative versus non-exudative age-related macular degeneration. Retina 1988;8:109-14. 
5 Holz FG, Wolfensberger TJ, Piguet B, et al. Bilateral macular drusen in age related macular degeneration. Prognosis and risk factors. Ophthalmology 1994;101:1522-8.

6 Abdelsalam A, Del Priore L, Zarbin MA. Drusen in age-related macular degeneration: pathogenesis, natural course, and laser photocoagulationinduced regression. Surv Ophthalmol 1999;44:1-29.

7 Little HL, Showman JM, Brown BW. A pilot randomized controlled study on the effect of laser photocoagulation of confluent soft macular drusen (see comments). Ophthalmology 1997; 104:623-31.

8 Frennesson IC, Nilsson SE. Effects of argon (green) laser treatment of soft drusen in early age-related maculopathy: a 6 month prospective study. Br J Ophthalmol 1995;79:905-9.

9 Bressler NM, Munoz B, Maguire MG, et al. Five-year incidence and disappearance of drusen and retinal pigment epithelial abnormalities. Waterman study. Arch Ophthalmol 1995;113:301-8.

10 Bressler SB, Bressler NM, Seddon JM, et al. Interobserver and intraobserver reliability in the clinical classification of drusen. Retina 1988;8:102-8.

11 Bird AC, Bressler NM, Bressler SB, et al. An international classification and grading system for age-related maculopathy and age-related macular degeneration. The International ARM Epidemiological Study Group. Surv Ophthalmol 1995;39:367-74.

12 Klein R, Davis MD, Magli YL, et al. The Wisconsin age-related maculopathy grading system. Ophthalmology 1991;98:1128-34.

13 G Age-Related Eye Disease Study Research. A randomized, placebocontrolled, clinical trial of high-dose supplementation with vitamins $C$ and $E$, beta carotene, and zinc for age-related macular degeneration and vision loss: AREDS report no. 8. Arch Ophthalmol 2001;119:1417-36.

14 Shin DS, Javornik NB, Berger JW. Computer-assisted, interactive fundus image processing for macular drusen quantitation (see comments). Ophthalmology 1999;106:1119-25.

15 Sebag M, Peli E, Lahav M. Image analysis of changes in drusen area. Acta Ophthalmologica 1991;69:603-10.

16 Morgan WH, Cooper RL, Constable IJ, et al. Automated extraction and quantification of macular drusen from fundal photographs. Aust N Z J Ophthalmol 1994;22:7-12.
17 Kirkpatrick JN, Spencer T, Manivannan A, et al. Quantitative image analysis of macular drusen from fundus photographs and scanning laser ophthalmoscope images. Eye 1995;9:48-55.

18 Peli PF, Lahav M. Drusen measurement from fundus photographs using computer image analysis. Ophthalmology 1986:93:1575-80.

19 Goldbaum MH, Katz NP, Nelson MR, et al. The discrimination of similarly colored objects in computer images of the ocular fundus. Invest Ophthalmol Vis Sci 1990;31:617-23.

20 Figueroa MS, Regueras A, Bertrand J, et al. Laser photocoagulation for macular soft drusen. Updated results. Retina 1997:17:378-84.

21 Figueroa MS, Regueras A, Bertrand J. Laser photocoagulation to treat macular soft drusen in age-related macular degeneration. Retina 1994;14:391-6.

22 J Sigelman. Foveal drusen resorption one year after perifoveal laser photocoagulation. Ophthalmology 1991;98:1379-83.

23 Neovascularization Prevention Trial Research Group. Laser treatment in eyes with large drusen. Short-term effects seen in a pilot randomized clinical trial. Choroidal Neovascularization Prevention Trial Research Group. Ophthalmology 1998:105:11-23.

24 Rapantzikos K, Zervakis M, Balas K. Detection and segmentation of drusen deposits on human retina: Potential in the diagnosis of age-related macular degeneration. Med Image Anal 2003;7:95-108.

25 Bone RA, Landrum JT, Cains A. Optical density spectra of the macular pigment in vivo and in vitro. Vision Res 1992;32:105-10.

26 Snodderly DM, Auran JD, Delori FC. The macular pigment. II. Spatial distribution in primate retinas. Invest Ophthalmol Vis Sci 1984;25:674-85.

27 Smith RT, Nagasaki T, Sparrow JR, et al. Patterns of reflectance in macular images: representation by a mathematical model. J Biomed Optics 2004:9:162-172

28 Smith RT, Nagasaki T, Sparrow JR, et al. A method of drusen measurement based on the geometry of fundus reflectance. Biomed Eng Online 2003;2:10.

29 Bland JM, Altman DG. Statistical methods for assessing agreement between two methods of clinical measurement. Lancet 1986;1:307-10.

30 Otsu N. A threshold selection method from gray-level histograms. IEEE Transactions on Systems, Man, and Cybernetics 1979;9:62-6. 